\title{
The Belle II Experiment
}

\author{
Matthew Barrett* \\ (On behalf of the Belle II collaboration) \\ University of Hawai' $i$ at Mānoa \\ E-mail: mattb@phys . hawaii.edu
}

The Belle II experiment is an upgrade to the Belle experiment, with a projected luminosity forty times that of its predecessor, allowing for precision measurements and the potential for new physics discoveries in the flavor sector. This document describes some of the physics that will be explored at Belle II, together with details of the construction and schedule of the experiment.

Flavor Physics \& CP Violation 2015

25-29 May 2015

Nagoya, Japan

${ }^{*}$ Speaker. 


\section{The $B$ factories}

The Belle and BaBar experiments, located at the KEKB and PEP-II accelerator rings respectively, have conducted a very successful physics programme since 1999. Together they collected over $1.5 \mathrm{ab}^{-1}$ of data, yielding a plethora of new physics results; the highlight of these was the experimental confirmation of the KM mechanism, that lead to the award of a share of the 2008 Nobel prize for Physics to M. Kobayashi and T. Maskawa.

Other physics highlights included the measurement of the parameters of the Unitarity triangle, and related parameters of the CKM matrix; the observation of mixing in $D$ mesons; observation of new $(X, Y, Z)$ hadrons; and the observation of direct $C P$-violation in $B$ meson decays. Many constraints were placed on New Physics, such as in the processes $B \rightarrow \tau \nu$ and $b \rightarrow s \gamma$.

In addition to being $B$ factories, KEKB and PEP-II were also excellent $\tau$ and charm factories, producing large numbers of these particles, allowing for the search for, for example, rare $\tau$ decays. Direct searches for new light particles, such as light Higgs bosons, or dark photons, were also undertaken with important limits placed in these searches.

\section{SuperKEKB}

SuperKEKB is an upgrade of the existing KEKB accelerator, with a design goal of an instantaneous luminosity forty times that of its predecessor. The beam currents in the accelerator will be double the currents used in KEKB, but the main increase in the luminosity comes from changes in the beamspot size from using nanobeams. Other upgrades include a new beam pipe, replacement of short dipoles with longer ones in the low energy ring, and new superconducting and permanent final focusing quadrupoles near the interaction point.

\section{Belle II}

Constructing the Belle II detector involves upgrading or replacing the all of the subdetectors of the Belle detector, to take advantages of advances in detector technology and to ensure that the new detector can operate in the increased luminosity environment provided by SuperKEKB.

A full description of the Belle II detector is available elsewhere [1]; some highlights of the design and recent construction are given here.

A new vertex detector will be installed, consisting of two layers of DEPFETs (Depleted Pchannel Field Effect Transistor) and four layers of Double Sided Silicon Detectors. The beam pipe radius has been reduced to $10 \mathrm{~mm}$, allowing the vertex detectors to be closer to the interaction point; the first DEPFET layer will have a radius of $14 \mathrm{~mm}$.

Outside the vertex detectors Belle II will have a new Central Drift Chamber (CDC), larger than the CDC of Belle. The stringing of the CDC for Belle II was completed in January 2014, with a total of 51,456 wires. Commissioning of the CDC is under way with cosmic rays, the first tracks from cosmic rays were seen in April 2015.

The particle identification system will have two completely new subdetectors: the (imaging) Time Of Propagation (TOP) subdetector for the barrel region, and an Aerogel Ring Imaging Cherenkov (ARICH) for the forward endcap. 
The TOP detector is made from sixteen top modules, each made from two quartz bars. An array of pixelated Micro-Channel Plate Photo Multiplier Tubes (MCP-PMT) is located at the backward end of the module; this detects Cherenkov photons created by the passage of charged particles through the quartz after the photons have propagated through the quartz bars via total internal reflection. Excellent spatial and temporal resolution will lead to excellent discrimination between the patterns of Cherenkov photons created by different types of charged particles. The first TOP modules for Belle II have been constructed, and commissioning of these modules with cosmic rays has started; each of the modules will be tested in this way prior to installation.

The ARICH has two layers of aerogel with different indices of refraction and an array of 420 hybrid avalanche photo detectors (HAPD). Cherenkov rings from photons created in the aerogel can be observed on the HAPDs; the two layers of aerogel lead to to a better photon yield, whilst maintaining resolution.

The electromagnetic calorimeter reuses Thallium doped Caesium Iodide crystals from Belle, but the readout system has had to be upgraded to accommodate the higher backgrounds expected in Belle II; the readout also includes waveform sampling electronics and additional features to improve performance.

The outer subdetector is the system for detecting $K_{L}$ and muons, named the KLM. The endcaps and sections of the barrel of the Belle KLM had to be replaced with new scintillators due to the increase in backgrounds expected. The Barrel KLM was the first subdetecor to be installed in Belle II, and commissioning has begun.

\section{Belle II Physics Prospects}

There are many potential signals of New Physics that could be seen at Belle II. These include:

- Flavour changing neutral currents;

- Lepton flavour violating decays;

- New Physics in $B \rightarrow \tau$ loops;

- Precision CKM measurements and new sources of CP-violation,

amongst many potential areas of investigation. In the flavour sector there will be different modes that will be favourable to search for in different experimental environments, and the searches that can be done at Belle II will be complementary with those of other experiments, such as LHCb.

\subsection{CKM parameters and CPV}

The B factories have greatly constrained the parameters of the Unitarity Triangle. New results from Belle II and $\mathrm{LHCb}$ will be able to further constrain, and over constrain the Unitarity Triangle.

For the angle $\phi_{1}(\equiv \beta)$ it is expected that an uncertainty on the quantity $\sin \left(2 \phi_{1}\right)$ of less than $1 \%$ can be achieved after several years of running of Belle II; this can be seen, for example, for the measurement of this quantity in the mode $B \rightarrow J / \psi K_{S}^{0}$ in Figure 1 . In this figure, and the following figure, the performance of Belle II in three different scenarios is shown: i) baseline Belle II performance with $70 \%$ of data recorded at a centre of mass energy corresponding to the mass of 
the $\Upsilon(4 S)$ resonance; ii) Belle II with improved $K_{S}^{0}$ performance; and iii) Belle II with improved $K_{S}^{0}$ performance and all data recorded at the $\Upsilon(4 S)$ resonance. These scenarios are also compared with projections of the measurement of this quantity at $\mathrm{LHCb}$, as described in Reference [2].

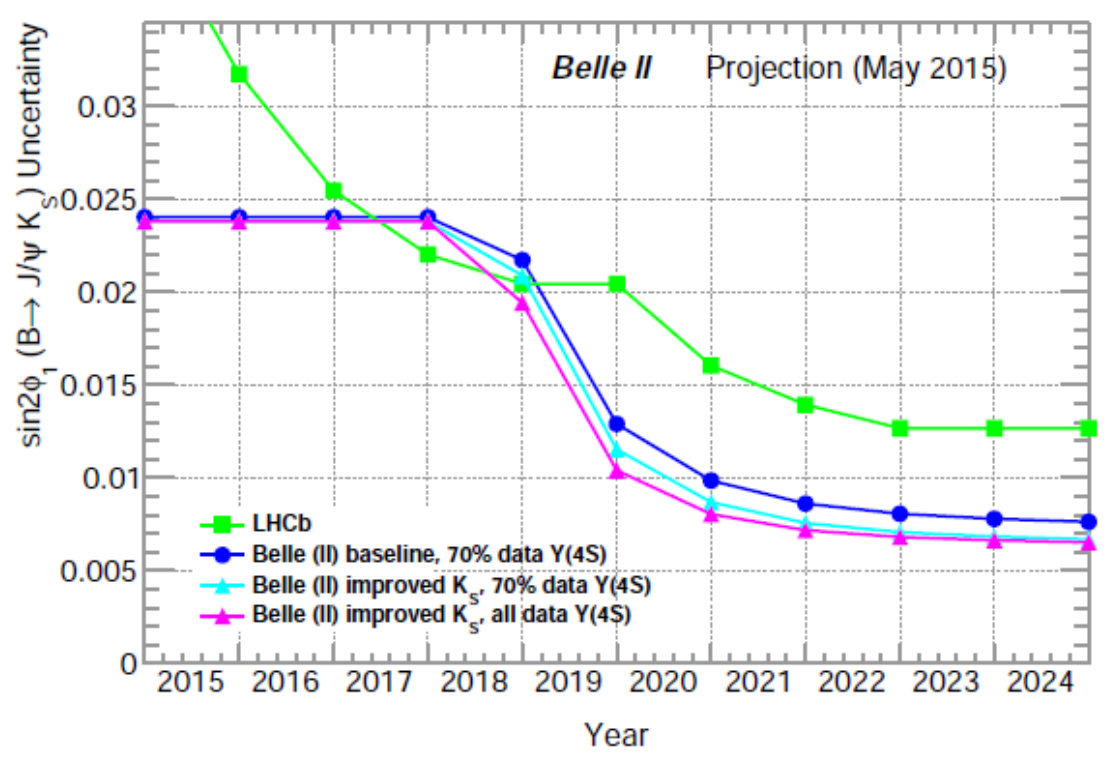

Figure 1: Projections for the uncertainty on the measurement of $\sin \left(2 \phi_{1}\right)$ for the mode $B \rightarrow J / \psi K_{S}^{0}$ for three different Belle II scenarios, and for LHCb [2].

An example of a set of modes where Direct CP-Violation measurements will be important is the $B \rightarrow K \pi$ modes. Different $A_{C P}$ values have been measured in different modes. A complete analysis will require all the different $B \rightarrow K \pi$ modes to be measured, including the modes containing neutral particles, such as $B \rightarrow K^{0} \pi^{0}$ where Belle II will have greater sensitivity. Figure 2 shows the projections of the uncertainty on $A_{C P}$ for the mode $B^{+} \rightarrow K_{S} \pi^{+}$; the scenarios shown are the same as those aforementioned.

\section{2 $B \rightarrow \tau \nu$ and $B \rightarrow D^{(*)} \tau \nu$}

In the decay $B \rightarrow \tau \nu$ there could be an enhancement to the branching fraction from the presence of a charged Higgs (replacing a $W$ boson as mediator for the decay). However this mode is experimentally challenging to measure due to the presence of two (or more) neutrinos in the final state, so $e^{+} e^{-}$colliders are preferable to hadron colliders. Current measurements have seen some evidence for this decay, but it has not yet been observed: evidence at $3.6 \sigma$ and $3.0 \sigma$ levels has been seen at Belle in a semileptonic tagged analysis [3] and a hadronic tagged analysis [4] respectively, while BaBar sees a $2.8 \sigma$ excess [5,6] (in each case $\sigma$ is the uncertainty of the respective measurement). Given this evidence $B \rightarrow \tau \nu$ is a mode that could potentially be observed from relatively early data at Belle II, and has the potential to put increasingly stringent constraints on New Physics. Figure 3 shows how the uncertainty on $B \rightarrow \tau \nu$ may improve with increasing integrated luminosity at Belle II. 


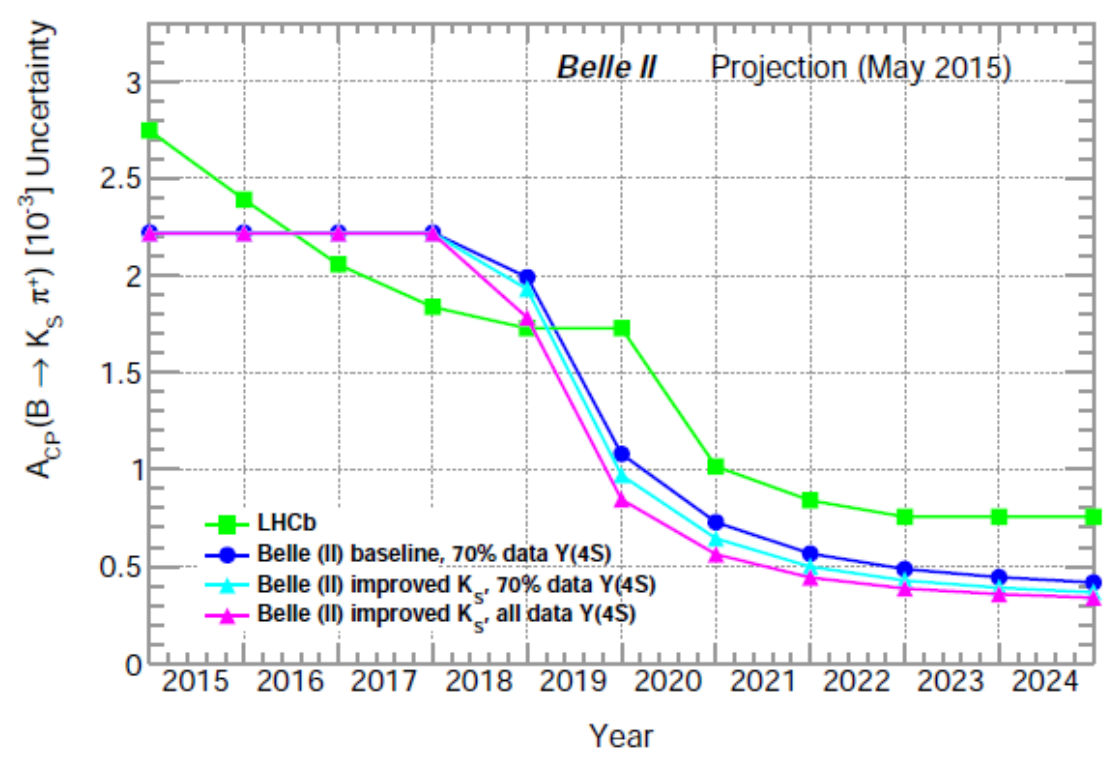

Figure 2: Projections for the uncertainty on the measurement of $A_{C P}$ for the mode $B^{+} \rightarrow K_{S} \pi^{+}$for three different Belle II scenarios, and for LHCb [2].

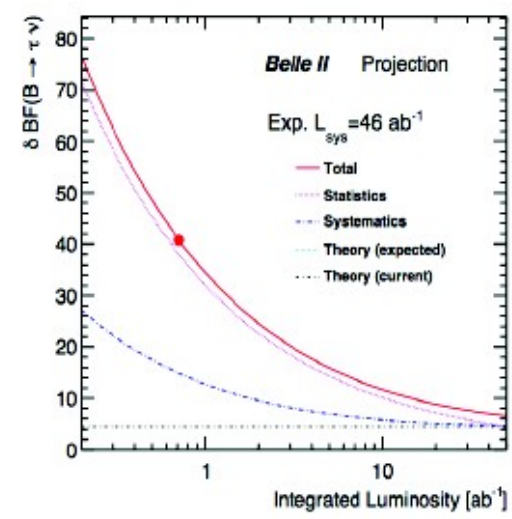

Figure 3: Projections for the uncertainty on the measurement of the branching fraction of $B \rightarrow \tau \nu$.

The modes $B \rightarrow D^{(*)} \tau \nu$ also have sensitivity to potential contributions from charged Higgs bosons. BaBar results [7] for these modes had a 3.4 $\sigma$ deviation from Standard Model predictions, and excluded the type II two Higgs Doublet model (2HDM) at a $99.8 \%$ confidence level. However new results were presented at this conference [8,9] from Belle $\left(B \rightarrow D^{*} \tau \nu\right.$ and $\left.B \rightarrow D \tau \nu\right)$ and LHCb $\left(B \rightarrow D^{*} \tau \nu\right)$, which are consistent with the Standard Model, and also with the BaBar result and the 2HDM. Belle II has the potential to resolve the current overlap between these measurements.

\section{3 $\tau$ Lepton Flavour Violation}

Lepton flavour violation (LFV) in the Standard Model is well below the sensitivity of experi- 
ments for the foreseeable future. Thus any observation of lepton flavour violation would be a clear and unequivocal observation of New Physics. There are a number of New Physics models that predict LFV in $\tau$ lepton decays with branching fractions as high as $10^{-10}$ to $10^{-7}$. Belle II could have sensitivity to observe or see evidence of these decay modes should this New Physics enhancement exist. Figure 4 shows the possible Belle II sensitivity for a number of $\tau$ LFV modes. For most of these modes the Belle II reach is obtained using a simple luminosity scaling from the Belle limits, which assumes that these modes are background free; this assumption is not valid for $\tau \rightarrow e \gamma$ and $\tau \rightarrow \mu \gamma$, and these projections include the projections of the expected backgrounds.

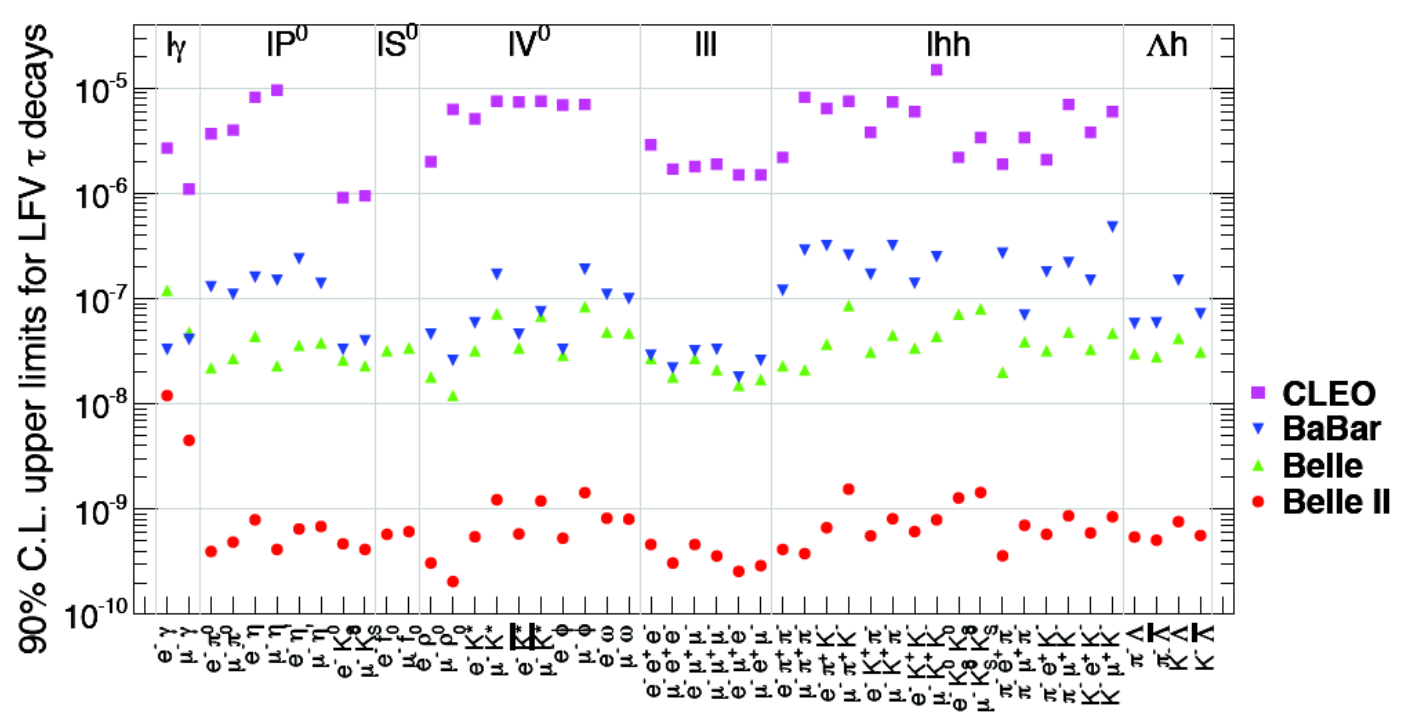

Figure 4: Current limits for selected lepton flavour violating $\tau$ lepton decays from the CLEO, BaBar, and Belle collaborations, and projections of expected limits for Belle II after collecting 50ab ${ }^{-1}$.

\section{Schedule and Commissioning}

Beams will start circulating in SuperKEKB in 2016. There will be three phases in the commissioning and operation of Belle II.

- Phase 1: SuperKEKB commissioning without Belle II. A commissioning detector will be used during this phase.

- Phase 2: Belle II will be rolled in, but without the inner vertex detectors.

- Phase 3: This phase will use the full Belle II detector proceeding to full operations.

Physics data taking with Belle II will start in 2018. The goal of Belle II is collect a data set with an integrated luminosity of $50 \mathrm{ab}^{-1}$. Figure 5 shows how the instantaneous and integrated luminosity of SuperKEKB/Belle II are projected to evolve over time. 
The commissioning detector, named BEAST II, used during Phases 1 and 2 will use several systems to measure the beam backgrounds before Belle II is fully installed. These will include MicroTPCs to measure fast neutron backgrounds, PIN diodes placed along the beam pipe to measure ionising particle backgrounds, calorimeter crystals, and slow neutron counters.

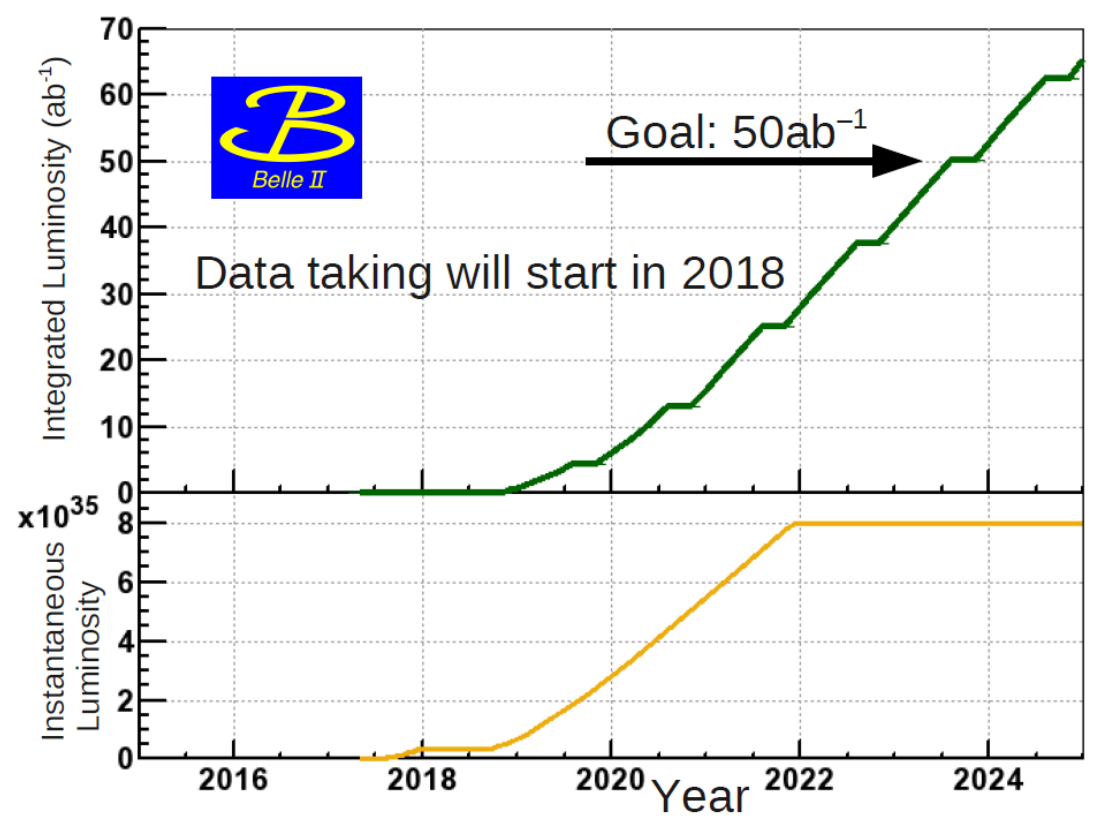

Figure 5: Instantaneous and Integrated Luminosity projections for Belle II.

\section{Summary}

These was, and indeed continues to be, a very rich and successful physics programme at the B-factories, offering hints of New Physics. To uncover these hints, and undertake a myriad of other studies, will require Belle II and complementary experiments. Belle II will start taking data in 2018 , and will aim to collect $50 \mathrm{ab}^{-1}$ of data, which will provide a much larger data set than the B factories; this will give greater sensitivity in many areas of flavour and CP physics and the potential for the observation of New Physics.

\section{References}

[1] Belle-II Collaboration (Abe, T. et al.) arXiv:1011.0352 [physics.ins-det].

[2] BELLE2-NOTE-PH-2015-02.

[3] K. Hara et al. (Belle Collaboration), PRD 82, 071101(R) (2010).

[4] K. Hara et al. (Belle Collaboration), PRL 110131801 (2013).

[5] B. Aubert et al. (BaBar Collaboration), PRD 77 011107(R) (2008). 
[6] B. Aubert et al. (BaBar Collaboration), PRD 81, 051101(R) (2010).

[7] J. P. Lees et al. (BaBar Collaboration), PRL 109, 101802 (2012).

[8] T. Kuhr, "New result $B \rightarrow D^{(*)} \tau \nu$ from Belle", PoS(FPCP2015)015 (this conference).

[9] G. Ciezarek, "New $\mathrm{D}(*)$ tau nu result from $\mathrm{LHCb}+$ non-B semileptonics", this conference. 